\title{
Brainhack: developing a culture of open, inclusive, community-driven neuroscience
}

Rémi Gau*1, Stephanie Noble ${ }^{\star 2}$, Katja Heuer ${ }^{\star 3,4}$, Katherine L. Bottenhorn ${ }^{\star 5}$, Isil P. Bilgin ${ }^{* 6,7}$, Yu-Fang Yang ${ }^{* 8}$, Julia M. Huntenburg ${ }^{* 9}$, Johanna Bayer ${ }^{* 10,11}$, Richard A.I. Bethlehem ${ }^{\star 12,13}$, Shawn A. Rhoads ${ }^{14}$, Christoph Vogelbacher ${ }^{15}$, Valentina Borghesani ${ }^{16}$, Elizabeth Levitis ${ }^{17,18}$, Hao-Ting Wang ${ }^{19,20,21}$, Sofie Van Den Bossche ${ }^{22}$, Xenia Kobeleva ${ }^{23,24}$, Jon Haitz Legarreta ${ }^{25}$, Samuel Guay ${ }^{26}$, Selim Melvin Atay ${ }^{27}$, Gael P. Varoquaux ${ }^{28,29}$, Dorien C. Huijser ${ }^{30,31}$, Malin S. Sandström ${ }^{32}$, Peer Herholz ${ }^{33}$, Samuel A. Nastase ${ }^{34}$, AmanPreet Badhwar ${ }^{35,16,36}$, Guillaume Dumas $^{37,38}$, Simon Schwab ${ }^{39}$, Stefano Moia ${ }^{40,41}$, Michael Dayan ${ }^{42}$, Yasmine Bassil ${ }^{43}$, Paula P. Brooks $^{34}$, Matteo Mancini ${ }^{20,44,45}$, James M. Shine ${ }^{46}$, David O'Connor ${ }^{47}$, Xihe Xie ${ }^{48}$, Davide Poggiali $^{49}$, Patrick Friedrich ${ }^{50}$, Anibal S. Heinsfeld ${ }^{54,55}$, Lydia Riedl ${ }^{51}$, Roberto Toro ${ }^{52,53}$, César Caballero-Gaudes ${ }^{40}$, Anders Eklund ${ }^{56,57,58}$, Kelly G. Garner ${ }^{59,60,61}$, Christopher R. Nolan ${ }^{62}$, Damion V. Demeter ${ }^{63}$, Fernando A. Barrios ${ }^{64}$, Junaid S. Merchant ${ }^{65,66}$, Elizabeth A. McDevitt ${ }^{34}$, Robert Oostenveld ${ }^{67,68}$, R. Cameron Craddock $^{69}$, Ariel Rokem ${ }^{70}$, Andrew Doyle ${ }^{71}$, Satrajit S. Ghosh ${ }^{72,73}$, Aki Nikolaidis ${ }^{74}$, Olivia W. Stanley ${ }^{75,76}$, Eneko Uruñuela ${ }^{40,41}$

\section{The Brainhack Community}

\section{The Brainhack Community:}

Nasim Anousheh ${ }^{77}$, Aurina Arnatkeviciute ${ }^{78}$, Guillaume Auzias ${ }^{79}$, Dipankar Bachar ${ }^{80}$, Elise Bannier $^{81}$, Ruggero Basanisi ${ }^{82}$, Arshitha Basavaraj ${ }^{83}$, Marco Bedini ${ }^{84}$, Pierre Bellec ${ }^{85,16}$, R. Austin Benn ${ }^{86}$, Kathryn Berluti ${ }^{14}$, Steffen Bollmann ${ }^{87,88}$, Saskia Bollmann ${ }^{88}$, Claire Bradley ${ }^{59}$, Jesse Brown ${ }^{89}$, Augusto Buchweitz ${ }^{90}$, Patrick Callahann ${ }^{91}$, Micaela Y. Chan ${ }^{92}$, Bramsh Q. Chandio $^{93}$, Theresa Cheng ${ }^{94}$, Sidhant Chopra ${ }^{95}$, Ai Wern Chung ${ }^{96}$, Thomas G. Close ${ }^{97,98}$, Etienne Combrisson ${ }^{99}$, Giorgia Cona ${ }^{100}$, R. Todd Constable ${ }^{101,102}$, Claire Cury ${ }^{103}$, Kamalaker Dadi $^{104}$, Pablo F. Damasceno ${ }^{105}$, Samir Das ${ }^{106}$, Fabrizio De Vico Fallani ${ }^{107,108}$, Krista DeStasio $^{109}$, Erin W. Dickie ${ }^{110,111}$, Lena Dorfschmidt ${ }^{13}$, Eugene P. Duff ${ }^{112}$, Elizabeth DuPre ${ }^{113}$, Sarah Dziura ${ }^{114}$, Nathalia B. Esper ${ }^{115}$, Oscar Esteban ${ }^{116}$, Shreyas Fadnavis ${ }^{117}$, Guillaume Flandin $^{118}$, Jessica E. Flannery ${ }^{119}$, John Flournoy ${ }^{120}$, Stephanie J. Forkel ${ }^{121,122}$, Alexandre R. Franco $^{123,74,124}$, Saampras Ganesan ${ }^{125,126}$, Siyuan Gao ${ }^{127}$, José C. García Alanis ${ }^{128}$, Eleftherios Garyfallidis $^{77}$, Tristan Glatard ${ }^{129}$, Enrico Glerean ${ }^{130,131}$, Javier Gonzalez-Castillo ${ }^{132}$, Cassandra D Gould van Praag ${ }^{133,134}$, Abigail S. Greene ${ }^{135}$, Geetika Gupta ${ }^{136,137}$, Catherine Alice Hahn ${ }^{138}$, Yaroslav O. Halchenko ${ }^{139}$, Daniel Handwerker ${ }^{140}$, Thomas S. Hartmann ${ }^{141}$, Valérie Hayot-Sasson ${ }^{129}$, Stephan Heunis ${ }^{142}$, Felix Hoffstaedter ${ }^{50,143}$, Daniela M Hohmann ${ }^{51}$, Corey Horien $^{144}$, Horea-loan loanas ${ }^{145,146}$, Alexandru lordan ${ }^{147}$, Chao Jiang ${ }^{148}$, Michael Joseph ${ }^{149}$, Jason Kai ${ }^{76}$, Agah Karakuzu ${ }^{150,151}$, David N. Kennedy ${ }^{152}$, Anisha Keshavan ${ }^{153,154,155}$, Ali R.' Khan $^{156}$, Gregory Kiar $^{29}$, P. Christiaan Klink ${ }^{157}$, Vincent Koppelmans ${ }^{158}$, Serge Koudoro ${ }^{117}$, Angela R. Laird ${ }^{159}$, Georg Langs ${ }^{160,161}$, Marissa Laws ${ }^{162}$, Roxane Licandro ${ }^{163,164}$, Sook-Lei Liew $^{165}$, Tomislav Lipic ${ }^{166}$, Krisanne Litinas ${ }^{167}$, Daniel J Lurie ${ }^{168}$, Désirée Lussier ${ }^{169}$, Christopher R. Madan ${ }^{170}$, Lea-Theresa Mais ${ }^{171}$, Sina Mansour L. ${ }^{126}$, J.P. Manzano-Patron ${ }^{172}$ ', Dimitra Maoutsa ${ }^{173}$, Matheus Marcon ${ }^{174}$, Daniel S. Margulies ${ }^{175,176}$, Giorgio Marinato ${ }^{177}$, Daniele Marinazzo ${ }^{178}$, Christopher J. Markiewicz ${ }^{179}$, Camille Maumet ${ }^{180}$, Felipe Meneguzzi ${ }^{174}$ ', David Meunier ${ }^{80}$, Michael P. Milham ${ }^{181}$, Kathryn L. Mills ${ }^{182,183}$, Davide Momi ${ }^{184}$, Clara A. Moreau $^{185,26}$, Aysha Motala ${ }^{186}$, Iska Moxon-Emre ${ }^{187}$, Thomas E. Nichols ${ }^{188,189}$, Dylan M. Nielson ${ }^{190}$, Gustav Nilsonne ${ }^{191,192}$, Lisa Novello ${ }^{193}$, Caroline O'Brien ${ }^{194}$, Emily Olafson ${ }^{48}$, Lindsay D. Oliver ${ }^{195}$, John A. Onofrey ${ }^{196}$, Edwina R. Orchard ${ }^{95}$, Kendra Oudyk ${ }^{197}$, Patrick J. Park $^{198}$, Mahboobeh Parsapoor ${ }^{199,200,201}$, Lorenzo Pasquini ${ }^{202}$, Scott Peltier ${ }^{167}$, Cyril R. 
Pernet $^{203}$, Rudolph Pienaar ${ }^{204,205}$, Pedro Pinheiro-Chagas ${ }^{206}$, Jean-Baptiste Poline ${ }^{207,208}$, Anqi $\mathrm{Qiu}^{209}$, Tiago Quendera ${ }^{210}$, Laura C. Rice ${ }^{211,212}$, Joscelin Rocha-Hidalgo ${ }^{14}$, Saige Rutherford $^{213}$, Mathias Scharinger ${ }^{214}$, Dustin Scheinost ${ }^{215}$, Deena Shariq ${ }^{216}$, Thomas B. Shaw $^{88}$, Viviana Siless ${ }^{217}$, Molly Simmonite ${ }^{218}$, Nikoloz Sirmpilatze ${ }^{219}$, Hayli Spence ${ }^{220}$, Julia Sprenger ${ }^{80}$, Andrija Stajduhar ${ }^{221,222}$, Martin Szinte ${ }^{80}$, Sylvain Takerkart ${ }^{80}$, Angela Tam ${ }^{223}$, Link Tejavibulya ${ }^{224}$, Michel Thiebaut de Schotten ${ }^{225,226}$, Ina Thome ${ }^{51}$, Laura Tomaz da Silva ${ }^{174}$, Nicolas Traut ${ }^{227,3}$, Lucina Q. Uddin ${ }^{228}$, Antonino Vallesi' ${ }^{229,230}$, John W. VanMeter ${ }^{231,232}$, Nandita Vijayakumar $^{233}$, Matteo Visconti di Oleggio Castello ${ }^{208}$, Jakub Vohryzek ${ }^{134}$, Jakša Vukojević $^{234}$, Kirstie Jane Whitaker ${ }^{235}$, Lucy Whitmore ${ }^{236}$, Steve Wideman ${ }^{237,238}$, Suzanne T. Witt ${ }^{239}$, Hua $X_{i e}^{66}$, Ting $X^{240}$, Chao-Gan Yan ${ }^{241,242}$, Fang-Cheng $Y^{243}{ }^{243}$, B.T. Thomas $Y_{e o^{244}}$, Xi-Nian Zuo $245,246,247$

Affiliations are listed at the end of this document.

\section{Author Contributions:}

The contributions of the different authors are detailed here, and our crediting system is described here.

* denotes equal contributions.

Corresponding Author and Lead Contact: +

\section{Abstract}

Brainhack is an innovative meeting format that promotes scientific collaboration and education in an open and inclusive environment. Departing from the formats of typical scientific workshops, these events are based on grassroots projects and training, and foster open and reproducible scientific practices. We describe here the multifaceted, lasting benefits of Brainhacks for individual participants, particularly early career researchers. We further highlight the unique contributions that Brainhacks can make to the research community, augmenting scientific progress by complementing opportunities available in conventional formats.

Keywords: best practices, Brainhack, collaboration, community building, hackathon, inclusivity, neuroscience, open science, reproducibility, training

Discipline: Life-science, Meta-science, Neuroscience (Cognitive Neuroscience, Computational Neuroscience, Systems Neuroscience, Other Neuroscience and Neurobiology) 


\section{Introduction}

Social factors play a crucial role in the advancement of science. New findings are discussed and theories emerge through social interactions, which usually take place within local research groups, and at academic events such as conferences, seminars or workshops. This system tends to amplify the voices of a select subset of the community-especially more established researchers-thus limiting opportunities for the larger community to contribute and connect. Brainhack events (or Brainhacks for short) complement these formats in neuroscience with decentralized two-to-five day gatherings, in which participants from diverse backgrounds and career stages collaborate and learn from each other in an informal setting. The Brainhack format was introduced in a previous publication (Craddock et al., 2016; Fig. 1A\&B). It is inspired by the hackathon ${ }^{12}$ model, which originated in software development and has gained traction in science as a way to bring people together for collaborative work and educational courses. Unlike many hackathons, Brainhacks welcome participants from all disciplines and with any level of experience-from those who have never written a line of code to software developers and expert neuroscientists. Brainhacks additionally replace the sometimes competitive context of traditional hackathons with a purely collaborative one, and also feature informal dissemination of ongoing research through unconferences ${ }^{3}$.

In the following, we aim to address two key questions about the merits of a Brainhack. First, how do participants benefit from attending a Brainhack event? Second, what is the relevance and importance of Brainhacks for neuroscience more broadly? To answer these questions, we discuss the five defining Brainhack features: 1) a Project-Oriented Approach that fosters active participation and community-driven problem-solving; 2) Learning By Doing, which enables participants to gain more intensive training, particularly in computational methods; 3 ) training in Open Science \& Collaborative Coding, which helps participants become more effective collaborators; 4) Focus on Reproducibility, which leads to more robust scientific research; and 5) accelerated Building and Bridging of Communities, which encourages inclusivity and seamless collaboration between researchers at different career stages. Altogether, Brainhacks and similar formats are increasingly recognized as a new way of providing academic training and conducting research that extends traditional settings. These events foster a new research culture that celebrates open science ${ }^{4}$, collaboration and diversity, unlocking opportunities for scientific progress.

\footnotetext{
1 "Hackathon" is a portmanteau of "hacking" and "marathon". Traditionally, it is an event where people and teams gather to collaboratively work on projects over the course of multiple days. These events often historically feature competitions between teams. Brainhacks instead emphasize collaboration over competition.

${ }^{2}$ Note that terms highlighted here are either defined in a unique way for this community or are important for appreciating the text. For detailed definitions, see Craddock et al., 2016, and the Brainhack Jupyter Book Glossary.

${ }^{3}$ An "unconference" refers to a short session in which participants present either their research or prompt a discourse on any topic of interest in an informal setting. The content of an unconference may be decided impromptu and is often inspired by ongoing team discussions during the course of the brainhack.

4 "Open Science" refers to a movement and practices within science aimed at increasing the transparency, accessibility, diversity, and inclusivity of scientific practices and output. This is often reflected in open science practices, such as publishing open access manuscripts, making research data Findable, Accessible, Interoperable, and Reusable (FAIR), open sourcing code and software, etc.
} 


\section{A Project-Oriented Approach}

Brainhacks are fundamentally centered around attendee-led projects. At the beginning of each Brainhack, participants pitch project ideas and form teams to realize some of these ideas during the "hacking" sessions (Fig. 1B). The teams are dynamic, and their composition can change throughout the course of a project. As participants group themselves based on their common interest in a question, method, goal or idea, interdisciplinary teams naturally emerge. Each participant can hone diverse skills by playing an active part in multiple projects. This format avoids the scientific silos that often arise when scientists connect over a specific methodological or conceptual approach. Brainhack projects promote the flow of information between specialized domains within the multidisciplinary field of neuroscience.

The project-oriented structure of Brainhack enables everyone to be an active participant at the event, with contributions taking a variety of forms. Importantly, the term "hacking" is not used to refer to coding in particular, but to describe an intensive form of work, eschewing strict conventions, and often targeted at prototyping an idea within a short period of time. Participants are therefore not required to have coding skills in order to make meaningful contributions. An example of an impactful project that did not focus on coding is Open Brain Consent (Open Brain Consent working group, 2021). This project developed consent form templates for the collection and sharing of human neuroimaging data, incorporating data protection standards such as the General Data Protection Regulation (GDPR) of the European Union. The consent forms can be used in ethics approval procedures to ensure that the collected data is shareable, while the participants' privacy is protected.

Unlike some traditional hackathons, there is no competitive element to Brainhacks. The focus is on collective and community-driven work, making the events more welcoming for inexperienced participants. Neither the level of completeness nor the publication potential determine the success of a project. Instead, Brainhacks emphasize the value of collaborating, exploring unconventional ideas, group thinking and building tools that benefit the community. Exemplary of these values is a project that originated at the first Brainhack in 2012: The Brain Catalogue provides magnetic resonance (MR) brain images of a range of different species and allows multiple users to view and segment them on the web simultaneously. Its successor, BrainBox, has evolved to enable real-time collaborative segmentation of any MR image accessible online (Heuer et al., 2016). BrainBox has been used in many subsequent Brainhack projects, research collaborations, and science outreach events. Similarly, braindr fosters citizen science while solving visual quality control for massive datasets (Keshavan et al., 2019). This app enables anyone to contribute to scientific progress by swiping left or right on brain images to classify them as clean or corrupted. The project originated from a hackathon in 2017, was extended in a Brainhack project in 2018, and recently led to the development of the extensible SwipesforScience citizen science template.

Many Brainhack projects take on a life of their own and grow beyond a single event. The open science approach embraced by the Brainhack community makes it easier for anyone to contribute to, or take the lead in pushing projects forward beyond their initial creation. For example, the Autism Gradients project, exploring the cortical hierarchy in individuals with 
autism, was conceptualised at Brainhack Global 2016. It was subsequently picked up by another group who expanded the original idea and invited the initial Brainhack team to collaborate. This resulted in a peer-reviewed publication (Hong et al., 2019), multiple follow-up projects, two exchange grants, and international workshops (Autism workshop at INSAR and gradient workshop at OHBM) on the same topic. Another example is the development of Nighres, a Python package for processing high-resolution neuroimaging data. The initial project spanned two Brainhacks in 2016, and resulted in a toolbox that made algorithms for layer-specific analysis of the cortex easier to install and use. This sparked the development of the full Nighres package, with a broader range of functions and various contributors across several Brainhacks. Nighres has been presented in a peer-reviewed publication (Huntenburg et al., 2018), is actively maintained, and has been used and cited in multiple studies. Some projects transcend the domain of brain sciences; for example, DueCredit is a project promoting citable code that emerged at Brainhack OHBM 2015 and is now used in molecular dynamics, geophysics, and other sciences. Many more projects have been part of Brainhacks over the years, attracting users and developers and evolving together with the community. An expanding list lives in the accompanying Jupyter Book.

All these examples highlight how the project-oriented approach of Brainhacks encourages active participation and interdisciplinary collaboration that can reach beyond a single event. The projects directly benefit participants, who can explore new ideas in a stimulating setting, leverage their projects for career advancement, and gain new skills by collaborating with experienced researchers and developers. Brainhack projects also contribute to the progress of the wider neuroscience community by fostering exchange across scientific silos, resulting in multi-disciplinary tools, community-driven guidelines and reference data, as well as traditional publication output.

\section{Learning by Doing}

Alongside projects, educational activities lie at the heart of Brainhacks. Such activities include informal teaching between project teammates, theoretical discussions in self-organized groups, unconference presentations, and structured workshops on a particular tool or topic. A recent format for major Brainhack events is the TrainTrack ${ }^{5}$, entirely education-focused sessions that run in parallel with project work (Fig. 1A-B). This format lowers the entry barrier for new participants, enabling them to build relevant skills and familiarize themselves with the structure and environment of a Brainhack before diving into their first project. The variety of educational approaches supports different ways of learning. Furthermore, the informal nature of these activities empowers participants to be proactive about learning and asking for help.

Brainhack instructors strive to share their materials with the scientific community, including recorded presentations, slide decks, or interactive tutorials. For example, all the materials developed for the TrainTrack of OHBM Brainhack 2020 have been made publicly available under a permissive license so as to encourage reuse, redistribution, and reproduction of the

\footnotetext{
${ }^{5} \mathrm{~A}$ "TrainTrack" is a series of educational workshops that run in parallel with the projects enabling attendees to acquire specific skills during the course of their projects. The content of a TrainTrack ranges from tutorials teaching skills useful to successfully navigate diverse projects in the Brainhack (such as code version control using Git) to more generalized education relevant to neuroscientific research (like MATLAB, BIDS, etc.).
} 
content. Educational content developed for Brainhacks covers a range of topics including analytical and statistical methods (e.g., machine learning, data preprocessing), reproducible workflows (e.g., automated pipelines, automated data standardization, version control, software containers), and other relevant concepts (e.g., preregistration, p-hacking). Brainhacks represent an ideal place to showcase neuroscientific tools in the form of presentations or training sessions. These sessions are designed to be hands-on and interactive as they typically feature small groups having active discussions. Participants are explicitly encouraged to adopt what they learned at a Brainhack event to their own context and to improve the teaching material with their own ideas.

Skills learned at Brainhacks are not constrained to those of a technical nature; the event format provides a unique opportunity for early career researchers to develop transferable skills such as teamwork and leadership. Project teams are often interdisciplinary, allowing participants to practice communicating beyond their own field. Everyone is encouraged to propose and lead their own projects, and the informal structure of the events often empowers more junior participants to also take on a leadership role. The growing pool of training materials provides a ready route to extend teaching opportunities to any member of the community, including trainees. Such experiences are rare for early career researchers, but crucial for their advancement given that they can potentially mold future interests, boost the quality of their research, and widen their scientific horizons. Altogether, the broad range of scientific and professional training opportunities equips participants with a skill set that can be applicable across many domains and career stages and may therefore open up a greater range of career opportunities.

\section{Open Science \& Collaborative Coding}

Despite the increasingly central role of programming in neuroscience research, formal training in coding is not common in the neuroscience curriculum. In addition, code is seldom shared across more than a few labs, and too often read and executed by only a single individual. As a result, many scripts and workflows are hard to reuse and share and may contain undiscovered errors (Merali et al., 2010).

By putting cross-disciplinary collaboration at its heart, Brainhacks have brought awareness to the need for usability, reusability, and long-term maintenance of tools. This comes with a shift of efforts, from individuals creating tools for their own needs to a community actively contributing to an existing resource, solving the aforementioned issues. Practices such as writing good code and documentation, improving code readability, performing basic version control, working collaboratively on a codebase on GitHub, GitLab, or BitBucket, and using appropriate open licenses have become essential within the community. These open practices and tools facilitate community-driven development and ensure that tools are available to all researchers, fostering global inclusivity. Brainhacks have highlighted the utility of producing a variety of research deliverables other than scientific papers (such as software, tutorials, workflows, and datasets), a concept that is increasingly endorsed by publishing venues such as $\underline{\mathrm{F} 1000}, \underline{\mathrm{RIO}}, \underline{\text { eLife}}, \underline{\text { Aperture, }}$, and others over the years.

Mastering collaborative programming skills enables Brainhack participants to contribute to open research objects which impact the wider scientific community. It can also make them 
more efficient at conducting their own research; for example, skills such as version control can be transferred to their own research group and foster more seamless collaboration amongst lab members. The wider neuroscience community benefits from the creation of transparent, reproducible tools, and from researchers equipped with the skills to maintain and extend them.

\section{A Focus on Reproducibility}

In line with their open, transparent, and collaborative nature, Brainhacks promote increased awareness of the importance of reproducible practices that integrate easily into research workflows. In addition to the coding practices mentioned above, an important aspect of reproducibility is data sharing. Public datasets are featured extensively in Brainhack projects and training sessions, since they are ideal for testing out new ideas or learning how to use a new tool. First-time users thus experiment with these datasets and related tools under the guidance of expert users, which lowers the barrier to working with public data in the future. This approach establishes open data sharing as a standard practice and teaches participants how to curate their own data and metadata to make them accessible and reusable by others.

Resources that help researchers handle their data in a reproducible fashion are integral to Brainhacks; many of these have been introduced to the Brainhack community through structured efforts from the Center for Reproducible Neuroimaging Computation (Kennedy et al., 2019). For example, many projects and trainings use DataLad, a tool that not only lets participants version-control their own data, but also helps them find, access, share, and work with increasingly large publicly available datasets. Similarly, a growing number of projects build on the Brain Imaging Data Structure (BIDS, Gorgolewski et al., 2016), a community standard for the organization of brain imaging data and metadata founded with the International Neuroinformatics Coordinating Facility (INCF). Introducing participants to data standards, such as BIDS, in the environment of a Brainhack allows them to experience the benefits of a unified data organization and provides them with the skillset to use these formats in their own research. Additionally, past Brainhacks have highlighted best practices in neuroimaging data analysis as defined by the Committee on Best Practice in Data Analysis and Sharing (COBIDAS guidelines for MRI as well as for EEG and MEG). By creating a scientific culture around open and standardized data, metadata, and methods, as well as detailed documentation and reporting, Brainhacks promote fundamental building blocks of a more efficient and reliable scientific research process.

\section{Building and Bridging Communities}

All aspects of Brainhacks discussed above build upon an active commitment to a diverse, inclusive, and non-hierarchically organized community. This commitment has been formalized in a Code of Conduct that aims to ensure a safe and welcoming environment for participants from all backgrounds. The Code of Conduct is discussed the beginning of a Brainhack and adherence is monitored throughout the event. There have also been dedicated efforts to raise awareness about equity, diversity and inclusivity, such as a recent panel discussion at Brainhack Ontario 2020. While far from perfect or bias-free, we feel that the Brainhack community itself is continuously growing more diverse in terms of race, 
ethnicity, gender identity and expression, sexual orientation, career stage, and other aspects of personal background and identity. The enthusiastically inclusive culture helps members hold each other to a standard of mutual respect that empowers individuals from typically underrepresented groups to claim their space and take on central roles in the community.

Brainhacks are designed to promote intensive networking. The project-oriented and decentralized setting puts participants on an equal footing regardless of backgrounds and career stage. Unconferences provide a unique opportunity for people interested in the same topic to meet and discuss, sometimes sparking new collaborations. Working in small groups during projects, workshops, and unconferences over the course of several days encourages frequent interactions that often go deeper than relatively short encounters at traditional conferences. These interactions contribute to building lasting collaborations that bridge across disciplines, research contexts, career stages, and geographical borders. Sometimes they lead to job opportunities, grant proposals, new ideas, and new projects. Often they turn into friendships. We firmly believe that growing this diverse community and insisting on a culture of collaboration and inclusivity has untold benefits for the retention and well-being of all scientists doing brain research.

In addition to year-round locally organized Brainhacks, Brainhack Global has emerged as a major yearly initiative that has sparked numerous simultaneous events around the world (Fig. 1C-D). The focus on open collaboration through virtual spaces throughout the years meant that the community had the infrastructure, knowledge and motivation to go fully virtual in 2020, accommodating restrictions due to the COVID-19 pandemic, budget constraints, and increased awareness of the climate cost of travel. The general format of project-oriented, community-building events has gained traction in the field of neuroscience and beyond. Large initiatives such as the Human Brain Project, Neurodata Without Borders, and the Society for the Improvement of Psychological Science have also chosen hackathons as a primary work format. Summer schools like the ABCD-ReproNim course, NeuroHackademy, the ABCD Workshop and the Brainhack School are based on the same principles as the original Brainhack events. Brainhacks have been organized with other communities such as Network Neuroscience, thereby forming a bridge with those communities. Many brainhack community members also play active roles in like-minded initiatives such as the Open Science Special Interest Group of OHBM or Neuromatch Academy, amongst others. Thus, a community of individuals and practices has emerged that transports the benefits and values of the brainhack format far beyond any individual event or organization. Brainhacks are spreading to an increasingly wide community, as their non-hierarchical, self-organising structure enables individuals to organize a Brainhack anywhere in the world (Fig. 1C), while events are kept financially accessible. An online community with over 4000 members and 500 channels uses the Mattermost messaging platform to provide continuity across time and space. Posts range from questions about a specific resource, to job openings and discussions about research ethics; and anyone can join regardless of having attended a Brainhack. The community evolves with every new member and their ideas, and many members become local advocates for the principles of open and collaborative science in their home institutions. 


\section{Conclusion \& Future Directions}

Brainhacks complement traditional academic settings and offer additional opportunities for participants to achieve their scientific and professional goals. The focus on building a community that promotes open science and inclusivity has naturally led to better coding practices, more reproducible methods, accelerated knowledge dissemination and ample opportunities for collaboration. Brainhacks differ from many scientific meetings, as they are more project-oriented, less formal, and have broadened the notion of what constitutes successful outputs in science. Within neuroscience, Brainhacks have the potential to evolve beyond their initial focus on neuroimaging data and include more projects on theory, hardware, and different types of neural data. With a growing global community, and an iteratively improving format (Fig. 1D), Brainhacks provide a successful template that can be extended to other scientific fields. Nearly a decade of successful Brainhacks have already brought about positive change for individual researchers and the field as a whole, in the form of improved skills, reusable resources, new collaborations, and a diverse and inclusive community.

\section{Figures \& Tables}

A. Anatomy of a Brainhack

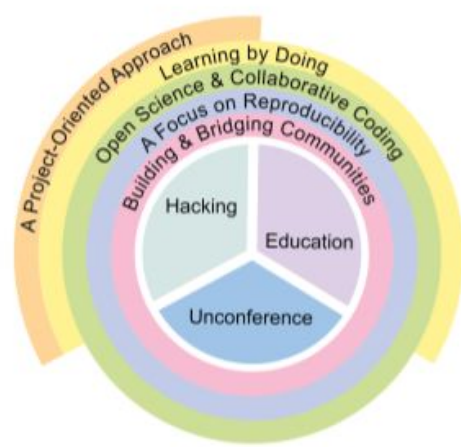

B. Timeline of a Single Event

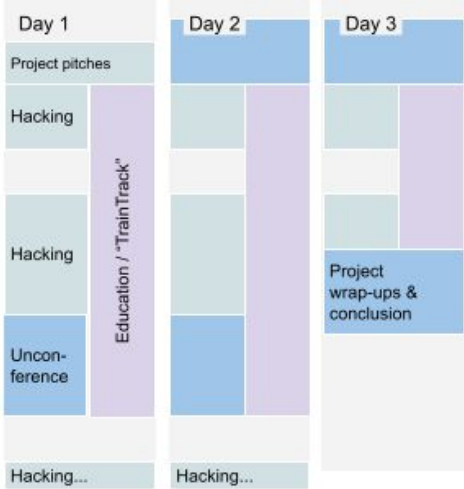

C. Brainhack Cartography
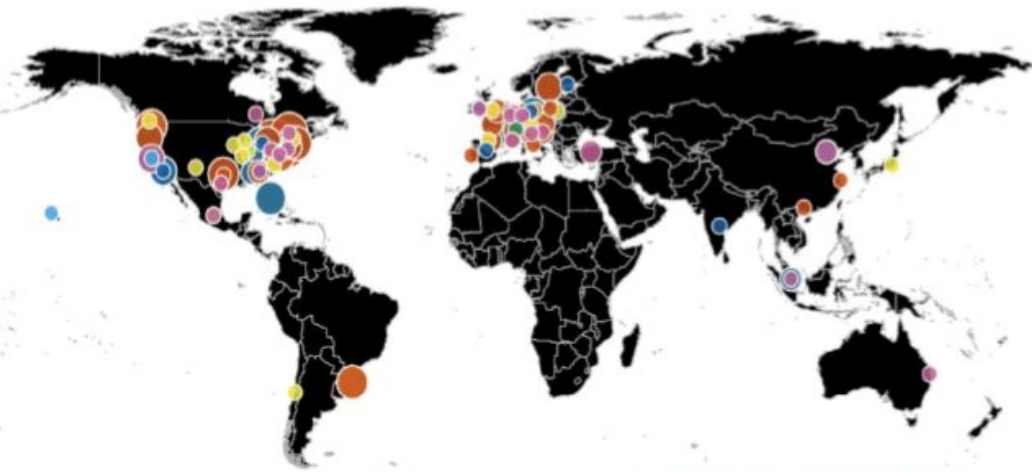

Number of events $10 \bigcirc \bigcirc \mathrm{O}$ Year $2012 \mathrm{OOO} 0 \mathrm{OO} 2020$

D. Brainhack Timeline

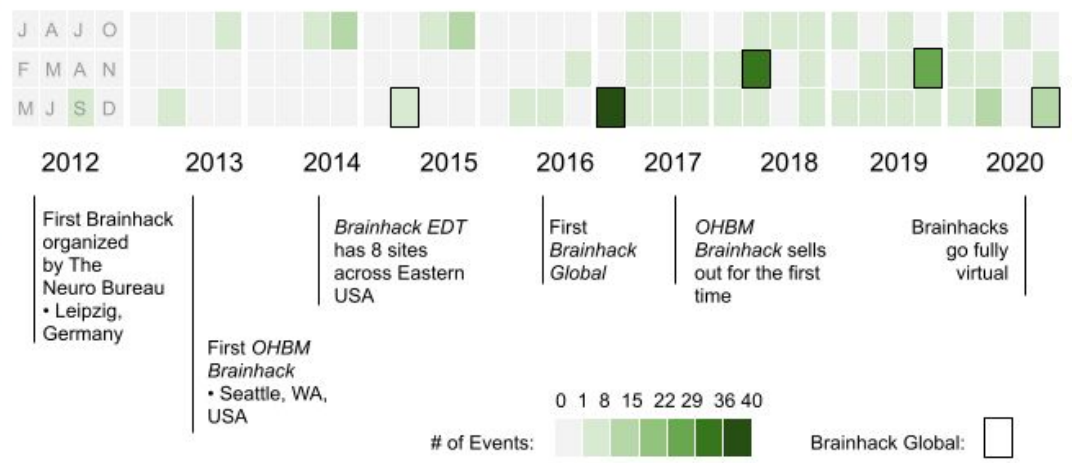

Figure 1. Brainhack in time and space. (A) Anatomy of a Brainhack shows how the components of Brainhack events relate to overarching topics that lead to scientific and professional opportunities in neuroscience. (B) Timeline of a Single Event describes the typical daily schedule throughout a brainhack. Project work and educational activities occur simultaneously interspersed with unconferences. (C) Brainhack Cartography displays cities 
across the world that have hosted a Brainhack. Marker color indicates the year in which each city hosted its first Brainhack, and marker size indicates the number of events hosted in each city. (D) The Brainhack Timeline displays the number of events per month since the inaugural Brainhack in September 2012, along with notable happenings throughout the years. Months are only denoted in the first year with a single letter; this ordering is repeated for all subsequent years. See the latest interactive version of this figure here.

\section{Acknowledgments}

The present manuscript is part of a growing community effort to collate BrainHack related insights and expertise into a Jupyter Book that will serve as a centralized set of resources for the community; we acknowledge all the individuals who contributed and will make ongoing contributions to these resources. The preprint of the present manuscript is available as part of the Jupyter Book here.

Moreover, we would like to acknowledge all Brainhack organizers, supporters, presenters, and participants for their contribution to growing and maintaining this community. The benefits described in this manuscript would not be possible without them. We also thank all institutions, labs, and organizations who have helped this community grow, meet in stimulating environments and add an excellent educational resource pool and agenda. With an expanding community, Brainhack's support network keeps growing and we thank all labs and individual researchers for their dedication and expertise offered to this community. These include (in alphabetical order): Allen Institute for Brain Science (Organization for Human Brain Mapping (OHBM) 2013), Amazon Web Services (OHBM 2013, OHBM 2014, Boston 2014, AMX 2015, Atlanta 2019), Athinoula A. Martinos Center for Biomedical Imaging (Boston 2014), Basque Center on Cognition, Brain and Language (Donostia 2018, 2019, 2020), Basque Government - Ikerbilerak grants (Donostia 2019), Biomedical Zone Ryerson University (Canadian Association for Neuroscience - Association Canadienne des Neurosciences (CAN-ACN) 2019), Brain Institute of Rio Grande do Sul (Porto Alegre 2016-2019), Brain Vision LLC (ATL 2019), Canadian Consortium on Neurodegeneration in Aging (Montreal 2017), Centre for Addiction and Mental Health (Global Toronto 2017-2019, Global Ontario 2020), Center for Advanced Brain Imaging (Atlanta 2019), Centre for Aging + Brain Health Innovation, Baycrest (Global Toronto 2019, Global Ontario 2020), Center for Intelligent Imaging (San Francisco 2019), Center for Translational Research in Neuroimaging \& Data Science (Atlanta 2019), Champalimaud Centre for the Unknown (Global Lisbon 2019), Child Mind Institute, Inc. (New York City 2014, Eastern Daylight Time 2014, Americas 2015, New York City 2015, Mexico 2015, Vienna 2016, Washington D.C. 2017, Global 2017, New York City 2017-2019, PRIMatE Data Exchange 2019), Canadian Open Neuroscience Platform (OHBM 2019, 2020; Global Montreal 2019, 2020, Global Toronto 2019, 2020, CAN-ACN 2019), Center for Open Neuroscience and Center for Cognitive Neuroscience at the Department of Psychological and Brain Sciences of Dartmouth College (Global Dartmouth 2017, 2018), Compute Ontario/SciNet (Global Toronto 2017, 2018, CAN-ACN 2019), Cognixion (CAN-ACN 2019), Donders Institute, the Netherlands (OpenMR Benelux 2020), Florida International University Center for Imaging Science (Miami EDT 2014, Miami Global 2017), ElementAl (Deep Brainhack 2017), Fetal-Neonatal Neuroimaging and Developmental Science Center, Boston Children's Hospital (Boston 2019), Frontiers (OHBM 2014), Frontiers in Neuroscience (OHBM 2013), 
George Washington Center for Healthcare Innovation and Policy Research (Washington D.C. 2017), Georgia State University Research Foundation (Atlanta 2019), Georgia Tech College of Sciences (Atlanta 2019), Georgetown University Center for Functional and Molecular Imaging (Washington D.C. 2019), Georgetown University Department of Psychology (Washington D.C. 2019, 2020), Georgetown University Methods Lab (Washington D.C. 2019, 2020), Healthy Brains for Healthy Lives (Deep Brainhack 2017), Gordon Museum of Pathology (Brainhack London 2017), Inria (Global Rennes 2018, Global Western France 2019), Intel Labs (Princeton 2019), International Neuroinformatics Coordinating Facility [INCF] (SfN 2012, ABA 2012, OHBM 2013-2016, OHBM 2020, Leiden 2014, Mexico 2015, NWB 2015, Neuroimaging Data Model 2015, 3xABBigNeuron 2015 (2xCambridge, 1xJanelia), ABBigNeuron 2016, Global Stockholm 2017-2019, Montreal 2018, NI 2019), INCF Belgian Node (Belgian Node Hackathon 2014), INCF Czech Node (Czech Node Hackathon 2019), INCF Japan Node (Brain Atlas Hackathon 2015, NIX\&odML Hackathon 2016-2018, 2020), Institut Pasteur (Paris 2016, Paris: Anatomy 2016, Global Paris 2017, 2018), Interaxon/Muse (CAN-ACN 2019, Global Toronto 2019), IRISA (Global Rennes 2018, Global Western France 2019), Karl-Franzens-Universtät Graz (Vienna 2016), La Paillasse (Global Paris 2017), Ludmer Centre for Neuroinformatics \& Mental Health (Open Science Hackathon 2019, OHBM 2018-2020; INCF 2018; Global Montreal 2017-2020), Marburg University (Marburg 2019, 2020), MATRICE (Paris 2013), Max Planck Institute for Cognitive and Brain Sciences (Leipzig 2012), McGill Centre for Integrative Neuroscience (Deep Brainhack 2017, Global 2017, OHBM 2018), McGill University, University of Montreal, Concordia University, Polytechnique Montreal (Montreal Brainhack School 2018-20), Microsoft Azure (OHBM 2015), Mozilla Lab Paris (Paris: Lesions 2017), National Institutes of Health (Washington D.C. 2017), NeuroMod (Global Montreal 2019; OHBM 2019, 2020), Neukom Institute (Global Dartmouth 2017, 2018), Neurohackademy (2016-2020), NeurotechX (Deep Brainhack 2017), NIH BD2K Center (1U54EB020406-01) Big Data for Discovery Science (USC, PI: Toga) (Los Angeles 2015, 2016), NIH BD2K Center (1U54EB020403-01) Enigma Center for Worldwide Medicine, Imaging, and Genomics (USC, PI: Thompson)(Los Angeles 2015, 2016), NIH BD2K Centers-Coordinating Center Hackathon Grant (Vienna 2016, Los Angeles 2016), NIH BD2K Supplement for NCANDA (3U01AA021697-04S1) and NCANDA: Data Analysis Component (5U01AA021697-04) (SRI International, PI: Pohl) (OHBM 2015, Mexico 2015), NVIDIA (Atlanta 2019), Ontario Brain Institute (Global Toronto 2017, 2019, CAN-ACN 2019, Global Ontario 2020), Opereason Analytics Inc. (Global Toronto 2019), Organization for Human Brain Mapping (OHBM 2013-2020), OHBM Australian chapter (OHBM 2020), OpenfMRI (OHBM 2016), OpenNeuro (OHBM 2020), Open Science Initiative University Marburg (Marburg 2018-2020), PNC - Padova Neuroscience Center (Padova 2018, 2019, 2020), Pontifical Catholic University of Rio Grande do Sul (Porto Alegre 2016-2019), Princeton University Department of Psychology Langfeld Fund (Princeton 2019), Quebec Bioimaging Network (Montreal 2014-15, 2017-19, OHBM 2020), ReproNim: Center for Reproducible Neuroimaging Computation (NIH-NIBIB P41 EB019936 (Los Angeles 2016, New York City 2020, Princeton 2019), Rotman Research Institute - Baycrest (Global Toronto 2019, Global Ontario 2020), Siemens (Paris 2013), Sticker Giant (Atlanta 2019), Toronto Neuroimaging Facility, Dept of Psychiatry, University of Toronto (Global Toronto 2019, Global Ontario 2020), Unité de Neuroimagerie Fonctionnelle, Centre de Recherche de I'Institut Universitaire de Gériatrie de Montréal (Montreal 2014-15), UNIQUE (OHBM 2020), University of Alabama at Birmingham (UAB 2017-2019), University of Miami Flipse Funds (Miami 2015), USC Viterbi School of Engineering (Los Angeles 2016), USC Stevens Neuroimaging and 
Informatics Institute (Los Angeles 2016), The University of Texas at Austin Dell Medical School (Global 2018), University of Warsaw (Warsaw 2017, 2019, 2020 (cancelled)), University of Zurich (Zurich 2018, 2019), Medical University of Vienna (Vienna 2016, 2019), Wellcome Centre for Integrative Neuroimaging (OHBM 2020), Wellcome Trust (OHBM 2016, Global Warwick 2017, PRIMatE Data Exchange 2019), WinAIML (Global Toronto 2019), Womanium (Washington D.C. 2017), The Neuro Bureau (Leipzig 2012, OHBM 2015, Washington D.C. 2017), University of Washington eScience Institute (Global 2016, 2019), and Yale University - MRRC and Biolmage Suite Web funds (Global 2018, 2019). An updated list of acknowledgments is available here.

\section{References}

Bannier, E., Barker, G., Borghesani, V., Broeckx, N., Clement, P., de la Iglesia Vaya, M., Emblem, K. E., Ghosh, S. S., Glerean, E., Gorgolewski, K. J., Havu, M., Halchenko, Y. O., Herholz, P., Hespel, A., Heunis, S., Hu, Y., Chuan-Peng, H., Huijser, D., Jancalek, R., ... Zhu, H. (2021). The Open Brain Consent: Informing research participants and obtaining consent to share brain imaging data. Human Brain Mapping; 1-10. https://doi.org/10.1002/hbm.25351

Craddock, R. C., Margulies, D. S., Bellec, P., Nichols, B. N., Alcauter, S., Barrios, F. A., Burnod, Y., Cannistraci, C. J., Cohen-Adad, J., De Leener, B., Dery, S., Downar, J., Dunlop, K., Franco, A. R., Froehlich, C. S., Gerber, A. J., Ghosh, S. S., Grabowski, T. J., Hill, S., ... $\mathrm{Xu}, \mathrm{T}$. (2016). Brainhack: a collaborative workshop for the open neuroscience community. GigaScience, 5(1). https://doi.org/10.1186/s13742-016-0121-x.

Gorgolewski, K. J., Auer, T., Calhoun, V. D., Craddock, R. C., Das, S., Duff, E. P., Flandin, G., Ghosh, S. S., Glatard, T., Halchenko, Y. O., Handwerker, D. A., Hanke, M., Keator, D., Li, X., Michael, Z., Maumet, C., Nichols, B. N., Nichols, T. E., Pellman, J., ... Poldrack, R. A. (2016). The brain imaging data structure, a format for organizing and describing outputs of neuroimaging experiments. Scientific Data, 3(1). https://doi.org/10.1038/sdata.2016.44.

Heuer, K., Ghosh, S., Robinson Sterling, A., \& Toro, R. (2016). Open Neuroimaging Laboratory. Research Ideas and Outcomes 2: e9113. https://doi.org/10.3897/rio.2.e9113

Hong, S.-J., Vos de Wael, R., Bethlehem, R. A. I., Lariviere, S., Paquola, C., Valk, S. L., Milham, M. P., Di Martino, A., Margulies, D. S., Smallwood, J., \& Bernhardt, B. C. (2019). Atypical functional connectome hierarchy in autism. Nature Communications, 10(1). https://doi.org/10.1038/s41467-019-08944-1

Huntenburg, J., Steele, C.J. \& Bazin P-L (2018). Nighres: processing tools for high-resolution neuroimaging. GigaScience, 7(7), https://doi.org/10.1093/gigascience/giy082

Kennedy, D. N., Abraham, S. A., Bates, J. F., Crowley, A., Ghosh, S., Gillespie, T., Goncalves, M., Grethe, J. S., Halchenko, Y. O., Hanke, M., Haselgrove, C., Hodge, S. M., Jarecka, D., Kaczmarzyk, J., Keator, D. B., Meyer, K., Martone, M. E., Padhy, S., Poline, J.-B., ... Travers, M. (2019). Everything Matters: The ReproNim Perspective on Reproducible Neuroimaging. Frontiers in Neuroinformatics, 13. https://doi.org/10.3389/fninf.2019.00001 
Keshavan, A., Yeatman, J. D., \& Rokem, A. (2019). Combining Citizen Science and Deep Learning to Amplify Expertise in Neuroimaging. Frontiers in Neuroinformatics, 13. https://doi.org/10.3389/fninf.2019.00029

Merali, Z. (2010). Computational science: ...Error. Nature 467, 775-777 https://doi.org/10.1038/467775a

\section{Affiliations}

${ }^{1}$ Institute of psychology, Université catholique de Louvain, Louvain la Neuve, Belgium, ${ }^{2}$ Radiology \& Biomedical Imaging, Yale University, New Haven CT, USA, ${ }^{3}$ Center for Research and Interdisciplinarity, Université Paris Descartes, Paris, France, ${ }^{4}$ Department of Neuropsychology, Max Planck Institute for Human Cognitive and Brain Sciences, Leipzig, Germany, ${ }^{5}$ Department of Psychology, Florida International University, Miami FL, USA, ${ }^{6}$ Biomedical Engineering, Cybernetics, University of Reading, Reading, UK, ${ }^{7}$ Allied Health Professions, University of the West of England, Bristol, UK, ${ }^{8}$ Department of Psychology, Würzburg University, Würzburg, Germany, ${ }^{9}$ Systems Neuroscience Lab, Champalimaud Research, Lisbon, Portugal, ${ }^{10}$ Faculty of Medicine, Dentistry and Health Sciences, University of Melbourne, Melbourne, Australia, ${ }^{11}$ Orygen Youth Health, Melbourne, Australia, ${ }^{12}$ Autism Research Centre, Department of Psychiatry, University of Cambridge, Cambridge, UK, ${ }^{13}$ Brain Mapping Unit, Department of Psychiatry, University of Cambridge, Cambridge, UK, ${ }^{14}$ Department of Psychology, Georgetown University, Washington D.C., USA, ${ }^{15}$ Laboratory for Multimodal Neuroimaging, Department of Psychiatry and Psychotherapy, University of Marburg, Marburg, Germany, ${ }^{16}$ Centre de Recherche de I'Institut Universitaire de Gériatrie de Montréal, University of Montréal, Montréal, Québec, Canada, ${ }^{17}$ National Institute of Mental Health, Bethesda MD, USA, ${ }^{18}$ Centre for Medical Image Computing, Department of Computer Science, University College London, London, UK, ${ }^{19}$ Sackler Centre for Consciousness Science, University of Sussex, Brighton, UK, ${ }^{20}$ Department of Neuroscience, Brighton and Sussex Medical School, University of Sussex, Brighton, UK, ${ }^{21}$ Sussex Neuroscience, University of Sussex, Brighton, UK, ${ }^{22}$ Department of Data Analysis, Faculty of Psychology and Educational Sciences, Ghent University, Ghent, Belgium, ${ }^{23}$ Department of Neurology, University of Bonn, Bonn, Germany, ${ }^{24}$ German Center for Neurodegenerative Diseases (DZNE), Bonn, Germany, ${ }^{25}$ Computer Science, Université de Sherbrooke, Sherbrooke, Canada, ${ }^{26}$ University of Montréal, Montréal, Québec, Canada, ${ }^{27}$ Neuroscience and Neurotechnology, Middle East Technical University, Ankara, Turkey, ${ }^{28}$ Parietal, Inria, Saclay, France, ${ }^{29}$ Montréal Neurological Institute, McGill University, Montréal, Québec, Canada, ${ }^{30}$ Erasmus School of Social and Behavioural Sciences, Erasmus University Rotterdam, Rotterdam, The Netherlands, ${ }^{31}$ Developmental and Educational Psychology, Leiden University, Leiden, The Netherlands, ${ }^{32}$ INCF, Karolinska Institute, Stockholm, Sweden, ${ }^{33}$ McConnell Brain Imaging Centre, The Neuro (Montreal Neurological Institute-Hospital), McGill University, Montréal, Québec, Canada, ${ }^{34}$ Princeton Neuroscience Institute, Princeton University, Princeton NJ, USA, ${ }^{35}$ Multiomics Investigation of Neurodegenerative Diseases (MIND) Lab, University of Montréal, Montréal, Québec, Canada, ${ }^{36}$ Département de pharmacologie et physiologie, University of Montréal, Montréal, Québec, Canada, ${ }^{37}$ Department of Psychiatry, University of Montréal, Montréal, Québec, Canada, ${ }^{38}$ Mila, University of Montréal, Montréal, Québec, Canada, ${ }^{39}$ University of Zurich, Zurich, Switzerland, ${ }^{40}$ Basque Center on Cognition, Brain and Language, San Sebastián-Donostia, Spain, ${ }^{41}$ University of the Basque Country (EHU UPV), San Sebastián-Donostia, Spain, ${ }^{42}$ Human Neuroscience Platform, Fondation Campus Biotech Geneva, Geneva, Switzerland, ${ }^{43}$ Graduate Division of Biological \& Biomedical Sciences, Emory University, Atlanta, USA, ${ }^{44}$ Cardiff University, Cardiff, UK, ${ }^{45}$ Polytechnique Montreal, Montréal, Québec, Canada, ${ }^{46}$ Faculty of Medicine and Health, The University of Sydney, 
Sydney, Australia, ${ }^{47}$ Department of Biomedical Engineering, Yale University, New Haven CT, USA, ${ }^{48}$ Department of Neuroscience, Weill Cornell Medicine, New York City, USA, ${ }^{49}$ Padova Neuroscience Center, University of Padova, Padova, Italy, ${ }^{50}$ Institute of Neuroscience and Medicine, Brain \& Behaviour (INM-7), Research Centre Jülich, Jülich, Germany, ${ }^{51}$ Department of Psychiatry and Psychotherapy, Philipps Universität, Marburg, Germany, ${ }^{52}$ Neuroscience department, Institut Pasteur, Paris, France, ${ }^{53}$ Center for Research and Interdisciplinarity (CRI), Université Paris Descartes, Paris, France, ${ }^{54}$ Computational Neuroimaging Lab, University of Texas at Austin, Austin TX, USA, ${ }^{55}$ Department of Computer Science, University of Texas at Austin, Austin TX, USA, ${ }^{56}$ Department of Biomedical Engineering, Linköping University, Linköping, Sweden, ${ }^{57}$ Department of Computer and Information Science, Linköping University, Linköping, Sweden, ${ }^{58}$ Center for Medical Image Science and Visualization (CMIV), Linköping University, Linköping, Sweden, ${ }^{59}$ Queensland Brain Institute, The University of Queensland, St Lucia, Australia, ${ }^{60}$ School of Psychology, University of Birmingham, Birmingham, UK, ${ }^{61}$ School of Psychology, The University of Queensland, St Lucia, Australia, ${ }^{62}$ Department of Psychology, University of New South Wales, Sydney, Australia, ${ }^{63}$ Psychology Department, The University of Texas at Austin, Austin TX, USA, ${ }^{64}$ Instituto de Neurobiología, Universidad Nacional Autónoma de México, Querétaro, México, ${ }^{65}$ Neuroscience and Cognitive Science Program, University of Maryland, College Park MD, USA, ${ }^{66}$ Department of Psychology, University of Maryland, College Park MD, USA, ${ }^{67}$ Donders Institute for Brain, Cognition and Behaviour, Radboud University, Nijmegen, The Netherlands, ${ }^{68}$ NatMEG, Karolinska Institutet, Stockholm, Sweden, ${ }^{69}$ Department of Diagnostic Medicine, The University of Texas at Austin Dell Medical School, Austin TX, USA, ${ }^{70}$ Psychology and eScience Institute, University of Washington, Seattle WA, USA, ${ }^{71}$ McGill Centre for Integrative Neuroscience, McGill University, Montréal, Québec, Canada, ${ }^{72}$ McGovern Institute for Brain Research, MIT, Cambridge, USA, ${ }^{73}$ Department of Otolaryngology - Head and Neck Surgery, Harvard Medical School, Boston, USA, ${ }^{74}$ Center for the Developing Brain, Child Mind Institute, New York City, USA, ${ }^{75}$ Centre for Functional and Metabolic Mapping, University of Western Ontario, London ON, Canada, ${ }^{76}$ Department of Medical Biophysics, University of Western Ontario, London ON, Canada, ${ }^{77}$ Intelligent Systems Engineering, Indiana University, Bloomington IN, USA, ${ }^{78}$ The Turner Institute for Brain and Mental Health, School of Psychological Sciences, Monash University, Victoria, Australia, ${ }^{79}$ Institut de Neurosciences de la Timone UMR 7289, Aix-Marseille Université, CNRS, ${ }^{80}$ Institut de Neurosciences de la Timone, CNRS - Aix-Marseille University, Marseille, France, ${ }^{81} \mathrm{CHU}$ Rennes, Radiology Department, Univ Rennes, CNRS, Inria, Inserm, IRISA UMR 6074, Empenn - ERL U 1228, F-35000 Rennes, France,, ${ }^{82}$ Institut de Neurosciences de la Timone UMR 7289, Aix Marseille Université, CNRS, Marseille, France,, ${ }^{83}$ National Institute of Mental Health,, ${ }^{84}$ Center for Mind/Brain Sciences, University of Trento,,${ }^{85}$ Psychology Department, University of Montréal, Montréal, Québec, Canada, ${ }^{86}$ Centro Nacional de Investigaciones Cardiovasculares (CNIC), Madrid, Spain, ${ }^{87}$ School of Information Technology and Electrical Engineering, The University of Queensland, Brisbane, Australia, ${ }^{88}$ Centre for Advanced Imaging, The University of Queensland, Brisbane, Australia, ${ }^{89}$ Department of Neurology, University of California San Francisco, San Francisco, USA, ${ }^{90}$ Brain Institute of Rio Grande do Sul, Pontifical Catholic University of Rio Grande do Sul, Porto Alegre, Brazil, ${ }^{91}$ Memory and Aging Center, UCSF,, ${ }^{92}$ Center for Vital Longevity, School of Behavioral and Brain Sciences, University of Texas at Dallas, Dallas TX, USA, ${ }^{93}$ Department of Intelligent Systems Engineering, Indiana University, Bloomington IN, USA, ${ }^{94}$ Department of Psychology, University of Oregon, USA,, ${ }^{95}$ Turner Institute for Brain and Mental Health, Monash University, Melbourne, Australia, ${ }^{96}$ Fetal-Neonatal Neuroimaging and Developmental Science Center, Boston Children's Hospital, Harvard Medical School, Boston, USA, ${ }^{97}$ School of Biomedical Engineering, The University of Sydney, Sydney, Australia, ${ }^{98}$ National Imaging Facility, Sydney, Australia, ${ }^{99}$ Aix-Marseille University, Institut de Neurosciences de la Timone,, ${ }^{100}$ Department of General Psychology, University of Padova, Padova, Italy, ${ }^{101}$ Yale MRRC, Yale University, New Haven CT, USA, ${ }^{102}$ School of Medicine, Yale University, New Haven CT, USA, ${ }^{103}$ Empenn ERL U 1228 IRISA UMR 6074, Univ Rennes, Inria, CNRS, 
Inserm, Rennes, France,, ${ }^{104}$ INRIA, ${ }^{105}$ Center for Intelligent Imaging, University of California, San Francisco, USA, ${ }^{106}$ McGill University,, ${ }^{107}$ ARAMIS team, Inria, Paris, France, ${ }^{108}$ Paris Brain Institute, Sorbonne Universites, Paris, France, ${ }^{109}$ University of Oregon,, ${ }^{110} \mathrm{Krembil}$ Centre from Neuroinformatics, Centre for Addiction and Mental Health, Toronto, Canada, ${ }^{111}$ Department of Psychiatry, University of Toronto, Toronto, Canada, ${ }^{112}$ University of Oxford, Oxford, UK, ${ }^{113}$ NeuroDataScience - ORIGAMI laboratory, McGill University, Montréal, Québec, Canada, ${ }^{114}$ Department of Psychology, University of Maryland, College Park, MD, USA,, ${ }^{115}$ Neuroimaging of Human Cognition, Brain Institute of Rio Grande do Sul, Porto Alegre, Brazil, ${ }^{116}$ Department of Radiology, Lausanne University Hospital, University of Lausanne, Lausanne, Switzerland, ${ }^{117}$ Indiana University, Bloomington IN, USA, ${ }^{118}$ Wellcome Centre for Human Neuroimaging, University College London, London, UK, ${ }^{119}$ University of North Carolina, Chapel Hill,, ${ }^{120}$ Department of Psychology, Harvard University, Cambridge, MA, USA,, ${ }^{121}$ CNRS UMR 5293 Institut des Maladies Neurodégénératives, Bordeaux, France, ${ }^{122}$ Department Neuroimaging, Institute of Psychiatry, Psychology and Neurosciences, King's College London, London, UK, ${ }^{123}$ Center for Biomedical Imaging and Neuromodulation, Nathan Kline Institute, Orangeburg NY, USA, ${ }^{124}$ Department of Psychiatry, NYU Grossman School of Medicine, New York City, USA, ${ }^{125}$ Systems Neuroscience, Melbourne Neuropsychiatry Centre, Melbourne, Australia, ${ }^{126}$ Department of Biomedical Engineering, The University of Melbourne, Melbourne, Australia, ${ }^{127}$ Yale University,, ${ }^{128}$ Department of Psychology, Philipps Universität, Marburg, Germany, ${ }^{129}$ Department of Computer Science and Software Engineering, Concordia University, Montréal, Québec, Canada, ${ }^{130}$ Department of Neuroscience and Biomedical Engineering, Aalto University, Espoo, Finland,, ${ }^{131}$ International Laboratory of Social Neurobiology, Institute of Cognitive Neuroscience, National Research University Higher School of Economics, Moscow, Russia, ${ }^{132} \mathrm{NIH},{ }^{133}$ Wellcome Centre for Integrative Neuroimaging, University of Oxford, Oxford, UK, ${ }^{134}$ Department of Psychiatry, University of Oxford, Oxford, UK, ${ }^{135}$ Interdepartmental Neuroscience Program and MD/PhD program, Yale School of Medicine, New Haven, CT, USA,, ${ }^{136}$ Department of Psychology, Western University, London ON, Canada, ${ }^{137}$ Brain and Mind Institute, Western University, London ON, Canada, ${ }^{138}$ Department of Radiology and Biomedical Imaging, Yale School of Medicine,, ${ }^{139}$ Center for Open Neuroscience, Department of Psychological and Brain Sciences, Dartmouth College, Hanover, NH, USA, ${ }^{140}$ Section on Functional Imaging Methods, National Institute of Mental Health, Bethesda, MD, USA,, ${ }^{141}$ Philipps Universität, Marburg, Germany, ${ }^{142}$ Department of Electrical Engineering, Eindhoven University of Technology, Eindhoven, The Netherlands, ${ }^{143}$ Institute of Systems Neuroscience, Medical Faculty, Heinrich-Heine University Duesseldorf, Duesseldorf, Germany, ${ }^{144}$ Interdepartmental Neuroscience Program, Yale School of Medicine, New Haven, CT, USA,, ${ }^{145}$ Biological Engineering, MIT, Cambridge, USA, ${ }^{146}$ D-ITET, ETH Zurich, Zurich, Switzerland, ${ }^{147}$ Department of Psychology, University of Michigan, Ann Arbor, MI, USA,, ${ }^{148}$ Key Laboratory of Behavioral Sciences, Institute of Psychology, Chinese Academy of Sciences, Beijing, China,, ${ }^{149}$ Campbell Family Mental Health Research Institute, The Centre for Addiction and Mental Health, Toronto, Canada, ${ }^{150}$ NeuroPoly Lab, Institute of Biomedical Engineering, Montréal, Québec, Canada, ${ }^{151}$ Montreal Heart Institute, University of Montréal, Montréal, Québec, Canada, ${ }^{152}$ University of Massachusetts Medical School,, ${ }^{153}$ Octave Bioscience, USA, ${ }^{154}$ eScience Institute, University of Washington, Seattle WA, USA, ${ }^{155}$ Institute for Learning and Brain Science, University of Washington, Seattle WA, USA, ${ }^{156}$ Robarts Research Institute, Western University, London, Canada, ${ }^{157}$ Department of Vision \& Cognition, Netherlands Institute for Neuroscience, Amsterdam, The Netherlands, ${ }^{158}$ Department of Psychiatry, University of Utah, Salt Lake City, UT, USA,, ${ }^{159}$ Department of Physics, Florida International University, Miami FL, USA, ${ }^{160} \mathrm{CIR}$ Department of Biomedical Imaging and Image-guided Therapy, Medical University of Vienna, Vienna, Austria, ${ }^{161} \mathrm{CSAIL}, \mathrm{MIT}$, Cambridge, USA, ${ }^{162}$ Center for the Study of Learning, Georgetown University Medical Center, Washington DC, USA, ${ }^{163}$ Medical University of Vienna, Vienna, Austria, ${ }^{164} \mathrm{TU}$ Wien, Vienna, Austria, ${ }^{165}$ Stevens Neuroimaging and Informatics Institute, University of Southern California, Los Angeles CA, USA, ${ }^{166}$ Laboratory for Machine Learning and Knowledge Representation, Ruder Boskovic 
Institute, Zagreb, Croatia, ${ }^{167}$ Functional MRI Laboratory, University of Michigan, Ann Arbor, USA, ${ }^{168}$ Department of Psychology, University of California, Berkeley, Berkeley, USA, ${ }^{169}$ Centre de recherche de I'Institut universitaire de gériatrie de Montréal,, ${ }^{170}$ Psychology, University of Nottingham, Nottingham, UK, ${ }^{171}$ University of Marburg, Marburg, Germany, ${ }^{172}$ Radiological Sciences \& Sir Peter Mansfield Imaging Centre, University of Nottingham, Nottingham, UK, ${ }^{173}$ Institute of Software Engineering and Theoretical Computer Science, Technical University of Berlin, Berlin, Germany, ${ }^{174}$ School of Technology, Pontifical Catholic University of Rio Grande do Sul, Porto Alegre, Brazil, ${ }^{175}$ Centre National de la Recherche Scientifique (CNRS), France, ${ }^{176}$ INCC UMR 8002, Université de Paris, Paris, France, ${ }^{177} \mathrm{CIMeC}$, University of Trento, Trento, Italy, ${ }^{178}$ Department of Data Analysis, Ghent University, Belgium,, ${ }^{179}$ Department of Psychology, Stanford University, Stanford, CA, USA,, ${ }^{180}$ Empenn ERL U 1228 IRISA UMR 6074, Univ Rennes, Inria, CNRS, Inserm, Rennes, France, ${ }^{181}$ Center for the Developing Brain, Child Mind Institute, New York, NY USA, ${ }^{182}$ Department of Psychology, University of Oregon, Eugene, USA, ${ }^{183}$ PROMENTA Research Center, Department of Psychology, University of Oslo, Oslo, Norway, ${ }^{184}$ Department of Neuroscience, Imaging and Clinical Sciences University "G. d'Annunzio" of Chieti Chieti Italy, ${ }^{185}$ Pasteur Institute, Paris, France, ${ }^{186}$ Department of Psychology, University of Western Ontario, London ON, Canada, ${ }^{187}$ Child, Youth and Family Service, Centre for Addiction and Mental Health, Toronto, Canada, ${ }^{188}$ Big Data Institute, University of Oxford, Oxford, UK, ${ }^{189}$ WIN-FMRIB, University of Oxford, Oxford, UK, ${ }^{190}$ Section on Clinical and Computational Psychiatry (Comp $\Psi$ ), National Institute of Mental Health, National Institutes of Health, Bethesda, Maryland,, ${ }^{191}$ Department of Clinical Neuroscience, Karolinska Institutet, Stockholm, Sweden, ${ }^{192}$ Department of Psychology, Stockholm University, Stockholm, Sweden, ${ }^{193}$ Center for Mind/Brain Sciences (CIMeC), University of Trento, Rovereto (Trento), Italy, ${ }^{194}$ Department of Neuroscience, Georgetown University, Washington, DC, United States,, ${ }^{195}$ Centre for Addiction and Mental Health, Toronto, Canada, ${ }^{196}$ Departments of Radiology \& Biomedical Imaging and of Urology, Yale School of Medicine, New Haven, USA, ${ }^{197}$ Montreal Neurological Institute, McGill University, Montreal, Canada, ${ }^{198}$ Engineering, University of Western Ontario, London ON, Canada, ${ }^{199}$ Computer Science Department, University of Toronto, Toronto, Canada, ${ }^{200}$ Vector Institute for Artificial Intelligence,, ${ }^{201}$ Women in Artificial Intelligence \& Machine Learning (WinAIML), ${ }^{202}$ Neurology Department, Memory and Aging Center, University of California San Francisco,, ${ }^{203}$ Centre for Clinical Brain Sciences, University of Edinburgh, Edinburgh, UK, ${ }^{204}$ Radiology, Boston Children's Hospital, Harvard Medical School, Boston, USA, ${ }^{205}$ Radiology, Harvard Medical School, Boston, USA, ${ }^{206}$ Stanford University,, ${ }^{207}$ Neurology and Neurosurgery, McGill University, Montréal, Québec, Canada, ${ }^{208} \mathrm{Helen}$ Wills Neuroscience Institute, University of California, Berkley, Berkeley, USA, ${ }^{209}$ Department of Biomedical Engineering, National University of Singapore, Singapore, Singapore, ${ }^{210}$ Champalimaud Research, Lisbon, Portugal, ${ }^{211}$ Neuroscience, American University, Washington D.C., USA, ${ }^{212}$ Neurology, Johns Hopkins University, Baltimore MD, USA, ${ }^{213}$ Department of Psychiatry, University of Michigan,, ${ }^{214}$ German Linguistics \& Fine Arts Science, Institute of German Linuguistics, University of Marburg, Marburg, Germany, ${ }^{215}$ Department of Radiology \& Biomedical Imaging, Yale School of Medicine,, ${ }^{216}$ University of Maryland, College Park,, ${ }^{217}$ Athinoula A. Martinos Center for Biomedical Imaging - Harvard Medical School - $\mathrm{MGH},{ }^{218}$ Department of Psychology, University of Michigan, Ann Arbor, USA, ${ }^{219}$ Functional Imaging Laboratory, German Primate Center - Leibniz Institute for Primate Research, Göttingen, Germany, ${ }^{220}$ Section on Molecular Neurobiology, National Institute of Child and Human Development, Bethesda, MD, ${ }^{221}$ Croatian Institute for Brain Research, School of Medicine, University of Zagreb, Zagreb, Croatia, ${ }^{222}$ Andrija Stampar School of Public Health, School of Medicine, University of Zagreb, Zagreb, Croatia, ${ }^{223}$ Perceiv Research Inc., Montréal, Québec, Canada, ${ }^{224}$ Interdepartmental Neuroscience Program, Yale University, New Haven CT, USA, ${ }^{225}$ Brain Connectivity and Behaviour Laboratory, Sorbonne Universities, Paris, France, ${ }^{226}$ Groupe d'Imagerie Neurofonctionnelle, Institut des Maladies Neurodégénératives- UMR 5293, CNRS, CEA University of Bordeaux, Bordeaux, France, ${ }^{227}$ Human Genetics and Cognitive Functions, Institut Pasteur, Paris, France, ${ }^{228}$ Department of Psychology, University of Miami, 
Florida, USA, ${ }^{229}$ Department of Neuroscience \& Padova Neuroscience Center, University of Padova, Padova, Italy, ${ }^{230}$ Brain Imaging and Neural Dynamics Research Group, IRCCS San Camillo Hospital, Venice, Italy, ${ }^{231}$ Department of Neurology, Georgetown University, Washington D.C., USA, ${ }^{232}$ Center for Functional and Molecular Imaging, Georgetown University Medical Center, Washington D.C., USA, ${ }^{233}$ School of Psychology, Deakin University, Melbourne, Australia, ${ }^{234}$ University psychiatric hospital Vrače, Zagreb, Croatia, ${ }^{235}$ Tools, Practices and Systems Research Programme, Alan Turing Institute, London, UK, ${ }^{236}$ Department of Psychology, University of Oregon, Eugene OR,, ${ }^{237} \mathrm{NBL}$, University of Alabama at Birmingham, Birmingham AL, USA, ${ }^{238} \mathrm{CNC}$, University of Alabama at Birmingham, Birmingham AL, USA, ${ }^{239}$ BrainsCAN, University of Western Ontario, London ON, Canada, ${ }^{240}$ Center for the Developing Brain, Child Mind Institute, New York, USA, ${ }^{241}$ CAS Key Laboratory of Behavioral Science, Institute of Psychology, Beijing, China, ${ }^{242}$ International Big-Data Center for Depression Research, Chinese Academy of Sciences, Beijing, China, ${ }^{243}$ University of Pittsburgh,, ${ }^{244}$ Department of Electrical and Computer Engineering, Centre for Sleep \& Cognition \& Centre for Translational MR Research, National University of Singapore, Singapore, Singapore,, ${ }^{245}$ State Key Laboratory of Cognitive Neuroscience and Learning, Beijing Normal University, Beijing, China, ${ }^{246}$ National Basic Public Science Data Center, Chinese Academy of Sciences, Beijing, China, ${ }^{247}$ School of Education Sciences, Nanning Normal University, Nanning, China

\section{Conflict of interest declaration}

\begin{tabular}{|l|l|}
\hline First name, Last Name & Conflict of interest \\
\hline Anisha Keshavan & Employee of Octave Bioscience \\
\hline
\end{tabular}

